International Conference KNOWLEDGE-BASED ORGANIZATION
Vol. XXVI

\title{
EXTENDING THE PRODUCTION TOLERANCE OF A GAUGE AND DETERMINING ITS TRUENESS
}

\author{
Danko TONEV, Branko SOTIROV \\ University of Rousse, Bulgaria \\ dtonev@uni-ruse.bg
}

\begin{abstract}
Gauges are widely used means for alternative control in mass production and, in some cases, in small-scale production. Similar to all measuring devices, their use is accompanied by the well-known errors Type I $(\alpha)$ and Type II ( $\beta)$. A basic requirement when designing gauges is that the values of these errors are equal to those of other measuring devices. Considering the development and improvement of technological processes, at present there is a real possibility to improve some of the gauges' characteristics. The most important metrological characteristic of the conical gauge is the socalled base distance. The standardized design requirements that are introduced to prevent scrap significantly reduce the production tolerance of the gauge. This not only causes some difficulties in the production of gauges, but also increases the likelihood of incorrectly rejecting conforming parts. The paper presents an opportunity to extend the production tolerance of conical gauges by applying a novel way of determining the nominal diameter of its no-go part, and by calculating theoretically the associated risk Type I $(\alpha)$.
\end{abstract}

Keywords: conical gauge, base distance, errors Type I ( $\alpha)$ and Type II ( $\beta$ )

\section{Introduction}

It is known that gauges are devices without a measurement scale that are widely used for alternative control. Meeting maximally Taylor's principle (principle of similarity), gauges allow fast and simple assessment of the complex geometrical accuracy of parts [4]. Even though they also possess many of the traditional metrological characteristics of measurement devices (MD), the control using gauges also is prone to errors Type I $(\alpha)$ and Type II $(\beta)$. A key requirement for designing gauges is that the values of these errors to match the permissible errors of the MD used to control a given object. It is known that a standard requirement for designing conical gauges is that the maximum length of the base distance prevents the exceeding of the maximum permissible value of the controlled dimension $\left(\mathrm{PHE}_{\max }\right)$.

This is why, aiming to prevent scrap, the length of the base distance is deliberately decreased by half the size of the tolerance when producing any of the two limit dimensions (sizes). This 'over-insurance' leads to decreasing the production tolerance of the gauge, and in some cases significantly hinders the 'mark-up' of both limit dimensions, additionally increases the percentage of wrongly scrapped good (conforming) parts and increases the value of $\alpha$. The improvement of the quality of control using conical gauges is associated with the need to decrease the risk Type I $(\alpha)$. 


\section{The problem}

The designing of conical gauges to some extent is similar to designing plain gauges where the main difference is the position of their 'No-Go' parts. It is known that for plain gauges it is possible to make a Type II $(\beta)$ error (a non-conforming dimension to be accepted as conforming), whereas for conical gauges this problem is eliminated by positioning the tolerance interval of its 'No-Go' part completely in the tolerance of the base diameter (Fig. 1). It is correct to note that the base fundamental deviation ' $Z$ ' for conical gauges to some extent

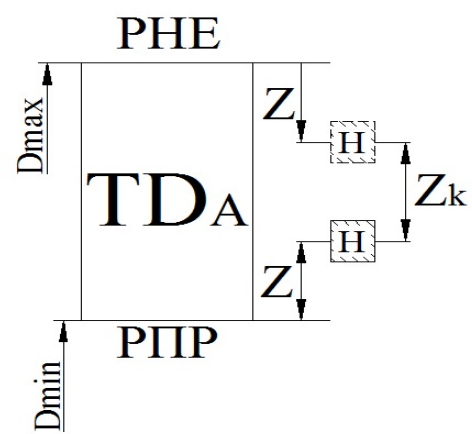

Figure 1: Principle scheme for designing of a conical plug gauge

compensates the form errors and the angular errors of the measured object. This positioning of the tolerance intervals enables the determining of some important characteristics of the gauge by applying one of the following two methods:

First method - differentiated determining of the two limit operational dimensions when designing the gauge using the dependencies:

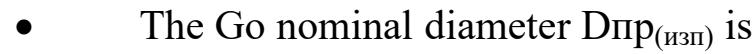
calculated using the dependency: Dпр (изп) $=$ $\mathrm{D}_{\min }+\mathrm{Z}$;

where, $D_{\min }$ is the smallest permissible dimension of the base diameter of the controlled hole, $\mathrm{z}$ is the coordinate (insert)

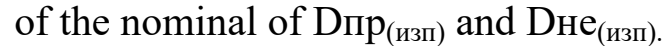

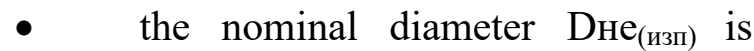
determined as Dне $_{(\text {изп) }}=\mathrm{D}_{\max }-\mathrm{Z}$.

where, $D_{\max }$ is the largest permissible dimension of the base diameter of the controlled hole.
In this manner, the base distance $Z_{k}$ is obtained as a dependent dimension. If this method is applied to a specific case, for example for designing a conical plug gauge for controlling a cone bushing with a base diameter $\varnothing 15 \mathrm{H} 12\left({ }^{+0,180}\right)$, length $\mathrm{L}=30$ $\mathrm{mm}$, taper conicity $\mathrm{C}=1: 7$ and angle $\alpha=8^{\circ} 10^{\prime} 16^{\prime \prime}$, made in AT $\alpha=9$, the resulting scheme is as presented in Fig. 2.

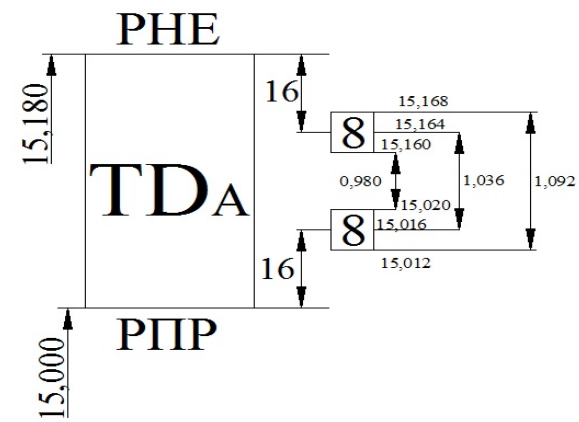

Figure 2: Scheme for a conical plug gauge $\varnothing 15 \mathrm{H12}\left(^{+0,180}\right)$

Second method (standardised)

The optimal variant for the dimensions of the operational diameters of the gauge, obtained according to the first method, is very rarely used in practice due to purely technological considerations. The standard procedure for designing a conical plug gauge, described in [1] recommends first to determine the 'Go' dimension Dпр (изп), and then the base distance between the two limit operational dimensions is calculated using the formula: $Z_{k}=\left(T D-2 Z-\frac{H}{2}\right) \cdot \frac{1}{C}$.

where, $\mathrm{H}$ is the production tolerance for limit operational dimensions of the gauge, and $\mathrm{C}$ is the conicity.

Finally, based on the 'dependent tolerances' it is easy to calculate the operational dimension of the 'No-Go' part of the gauge. In the same document it is presented by dash line (Fig. 1) in order to show that it is obtained as a dependent dimension [1].

When applying the two described methods it is natural to obtain different values of the base distance and the operational dimension of the 'No-Go' limit diameter. 
At the same time, there are objective possibilities for improving the manufacturability during the design and production of conical gauges.

Fig. 2 and Fig. 3 show the linear dimensions of the plug gauge obtained

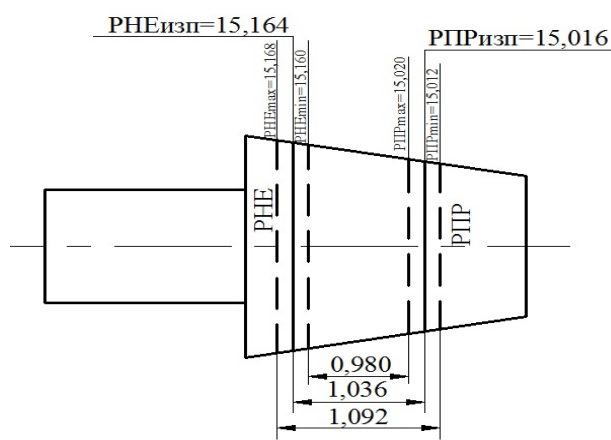

Figure 3: Scheme of a conical plug gauge $\varnothing 15 H 7\left(^{+0,180}\right)$

using the first method (Fig. 2), for which the optimal (and the nominal) base distance is $Z_{k}=1,036 \mathrm{~mm}$. As explained earlier, $Z_{k}$ is a 'dependent dimension' obtained from the previously known two limit dimensions. Their values vary within $\pm \frac{H}{2}$, which in turn leads to a variation in the values of the base distance. Therefore, the value of the base distance can be calculated using the following dependence

$Z_{k}=(T D-2 Z) \cdot \frac{1}{C} \pm \frac{H}{C}$

For the discussed example, its numeric value will be:

$$
\begin{aligned}
& Z_{k}=(0,18-2 \cdot 016) \cdot \frac{1}{(1: 7)} \pm \frac{0,008}{(1: 7)} \\
& Z_{k}=1,036 \pm 0,056
\end{aligned}
$$

As noted above (dependence 2), the standardized method for designing a plug gauge uses two parameters - Dпр (изп) the nominal value of the base distance $Z_{k}$. Both approaches exclude the possibility of error Type II ( $\beta)$. According to [2] the value of the base distance $Z_{k}$ is calculated using dependence:
$Z_{k}=\left(T D-2 Z-\frac{H}{2}\right) \cdot \frac{1}{C} \pm \frac{H}{2 C}$,

i.e. for the discussed example it results in:

$Z_{k}=\left(0,18-2 \cdot 0,016-\frac{0,008}{2}\right) \cdot \frac{1}{(1: 7)} \pm \frac{H}{2 \cdot(1: 7)}$

$Z_{k}=1,008 \pm 0,028$

The discussion above demonstrates that the value of the base distance $Z_{k}$ can be determined in a way which allows increasing the production tolerance and the manufacturability of the gauge while maintaining the remaining standard requirements for the gauge. It is obvious that the two design types will lead to different values for the base distance, and each approach has its natural advantages and disadvantages.

The aim of this paper is to study and to compare the proposed approach to the standard approach for designing conical gauges in relation to the probability to make Type I error $(\alpha)$.

\section{Comparison of the two approaches}

It is correct to start the competitive comparison, that aims to determine the probability to make Type I error $(\alpha)$ for both approaches, by determining the nominal operational dimension of the 'Go' part, which for the presented example is Dпр

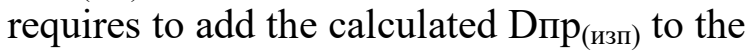
dimension of the base distance (with the relevant deviation) and using dependence:

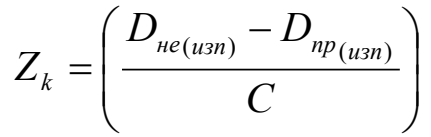

to determine the dimension of the 'No-Go' part $\mathrm{D}_{\text {не(изп) }}$.

By using dependence (4) it is easy to to obtain $Z_{\mathrm{k}}=1,008 \pm 0,028 \mathrm{~mm}$.

Naturally, the fundamental deviation of Dпр (изп) also varies within the limits of its tolerance interval (Fig. 2). 
The percentage of incorrectly scrapped parts is calculated according to a standard methodology [2]. It can be seen from the scheme of the transition fit, presented on Fig. 4 that the probability of occurrence of interference fits with interference $\mathrm{S}$ is bigger than the probability of occurrence of clearance fits with clearance J. For the presented example:

$\mathrm{S}_{\max }=164 \mu \mathrm{m}$;

$\mathrm{J}_{\max }=24 \mu \mathrm{m} \rightarrow \mathrm{Smin}=-24 \mu \mathrm{m}$;

$S m=\frac{S \max +S \min }{2}=\frac{164+(-24)}{2}=70 \mu \mathrm{m}$

and the total variation $\sigma_{\mathrm{S}}$ is:

$$
\sigma_{s}=\sqrt{\sigma_{A}+\sigma_{A r}}=\sqrt{30^{2}+1,33^{2}}=30,02 \mu m
$$

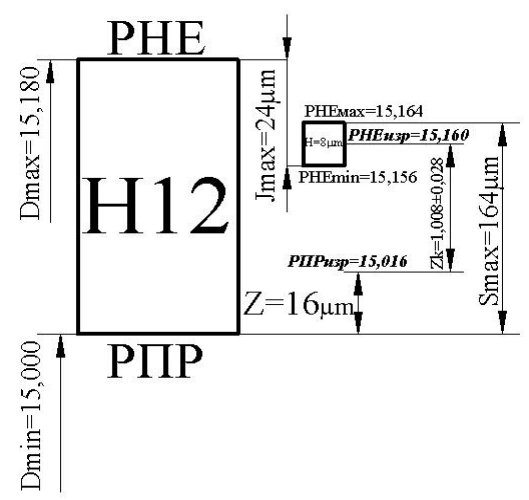

Figure 4: Scheme for determining error Type I

(a) for base distance $Z_{k}=1,008 \pm 0,028$ and

$$
z=16 \mu \mathrm{m}
$$

where, $\sigma_{\mathrm{A}}$ is the variation of the base diameter of the controlled part, $\sigma_{\mathrm{Ar}}$ the variation of the diameter of the 'No-Go' part of the conical gauge, $\mathrm{Sm}$ - average tightness.

Upon short calculations, the probabilities of occurrence of interference and clearance fits are determined:

$\Phi\left(\mathrm{t}_{1}\right)=\Phi(2,33)=0,49$;

$\Phi\left(\mathrm{t}_{2}\right)=\Phi(3,13)=0,4987$.

Consequently, the probability of obtaining an interference fit is:

$\mathrm{PS}=\Phi\left(\mathrm{t}_{1}\right)+\Phi\left(\mathrm{t}_{2}\right)==0,49+0,4987=$ $=0,9887 \rightarrow 98,87 \%$, and the probability of clearance fits is:

$\mathrm{PJ}=1-\mathrm{PS}=1-0,9887=0,0113 \rightarrow 1,13 \%$

Therefore, in $98,87 \%$ of the cases the gauge will differentiate correctly between the good (conforming) and the bad (nonconforming) parts, but in $1,13 \%$ of the cases there is a probability of occurrence of a 'false' signal.

Similarly, the same probabilities are determined when using the proposed new approach, presented in Section 2.

Fig. 5 shows the calculated dimensions of the gauge, and the values of the corresponding probabilities are:

$\mathrm{PS}=\Phi\left(\mathrm{t}_{1}\right)+\Phi\left(\mathrm{t}_{2}\right)=$

$0,493+0,4991=0,9924 \rightarrow 99,21 \%$

$\mathrm{PJ}=1-\mathrm{PS}=1-0,9921=0,0079 \rightarrow 0,79 \%$

The comparison of the results, obtained using the proposed new approach, and those from the standard approach, shows that the probability for the gauge to give a 'false' signal for the new approach is significantly lower: $-0,79 \%$ against $1,13 \%$. Comparative calculations for $\mathrm{z}=20 \mu \mathrm{m}$ and $\mathrm{z}=12 \mu \mathrm{m}$ are made in a similar way. Table 1 Results from the competitive comparison of the two approaches, presents the calculated numerical values of the risk $\alpha$ when using the compared two approaches for values of the base distance $Z_{k}=1.036 \mathrm{~mm}$ and $Z_{k}=$ 1.008 at $\mathrm{z}=12 \mu \mathrm{m}, \mathrm{z}=16 \mu \mathrm{m}$ and $\mathrm{z}=20 \mu \mathrm{m}$. The analysis of the calculated results that compare both methods for determining the diameter of the 'No-Go' part of the gauge,

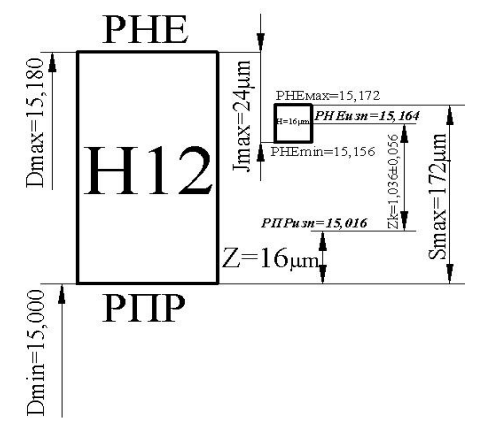

Figure 5: Scheme for determining error Type $I(\alpha)$ for base distance $Z_{k}=1,036 \pm 0,056$ at $z=16 \mu \mathrm{m}$ 
and the resulting incorrectly scrapped parts in percentage, shows that:

- $\quad$ for $z=16 \mu \mathrm{m}$ the value of the $\alpha$ error is $0,79 \%$ for the proposed new approach and $1,13 \%$ for the standard approach. The relative difference between the two values is $43 \%$;

- $\quad$ for $\mathrm{z}=20 \mu \mathrm{m}$ the value of the $\alpha$ error is $0,56 \%$ for the proposed new approach and $0.83 \%$ for the standard approach, and the relative difference between the two values is $48 \%$;
- $\quad$ for $z=12 \mu \mathrm{m}$ the value of the $\alpha$ error is $1,08 \%$ for the proposed new approach and $1,53 \%$ for the standard approach. The relative difference between the two values is $41 \%$;

Therefore, for each of the discussed cases, the value of the Type I error $(\alpha)$ made for the proposed new approach is always smaller by about $41 \%-48 \%$ of the Type I error $(\alpha)$, made when using the standard approach.

Table 1 Style and font size for printer-ready papers

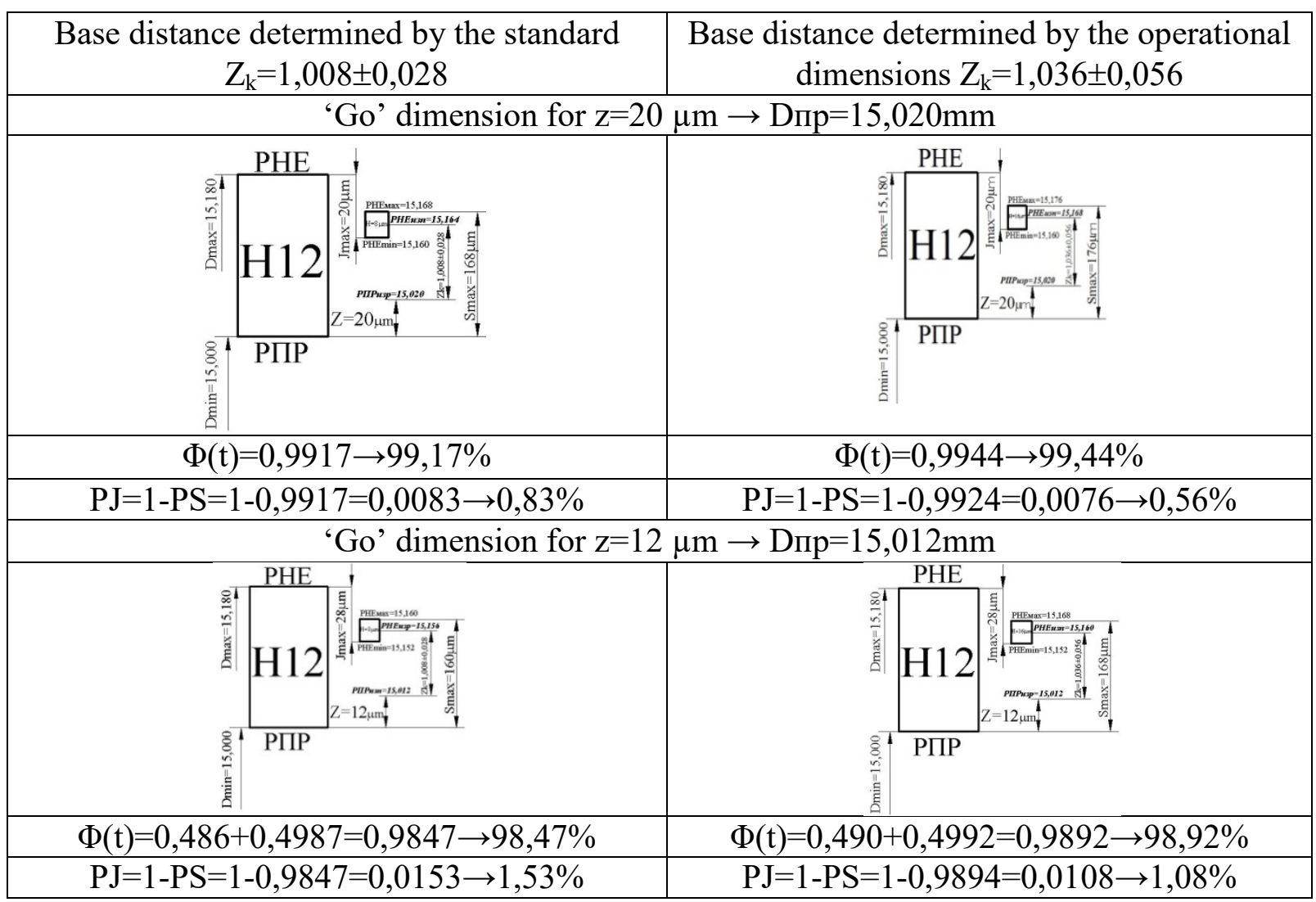

\section{Conclusions}

- A new approach is proposed for determining the value of the base distance of a conical gauge as a dependent dimension based on the independently determined two limit operational dimensions, leading to an increase of its production tolerance.

- A comparative analysis of the Type I error $(\alpha)$ which is made when using the standard and the proposed new design method proves that for identical control parameters the value of $\alpha$ for the new method is smaller.

- $\quad$ The proposed new design approach allows to improve the manufacturability in the production of conical gauges and to improve the quality of control with such gauges. 


\section{References}

[1] BDS 15650-83. Kalibri za gladki valove i otvori.

[2] Kutay, A, K., Sorochkin, B, M., Tochnost i proizvodstvenyy kontrol v mashinostroenii, Spravochnik, Leningrad "Mashinostroenie", 1983.

[3] Sakakushev B., S. Parvanov, Tz. Gueorguiev. The Real Capabilities of the Photogrammetric and the Stereophotogrammetric Methods for Measurement in Mechanical and Manufacturing Engineering. Proceedings of SOCIOINT 2018- 5th International Conference on Education, Social Sciences and Humanities, 2-4 July 2018Dubai, U.A.E., 2018, No 1, pp. 108-114, ISSN 978-605-82433-3-0.

[4] Tonev, D. Confedence of control using limit gauges for plain cylindrical products. XXI National scientific symposium with international participation Metrology and metrology assurance, Sozopol, 2011, pp. 300-305, ISSN 1313-9126. 